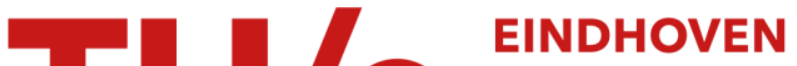 UNIVERSITY OF TECHNOLOGY
}

\section{Optimization of acoustic coupling for bottom actuated scattering based subsurface scanning probe microscopy}

\section{Citation for published version (APA):}

van Neer, P. L. M. J., Quesson, B., van Riel, M., van Es, M. H., Hatakeyama, K., Mohtashami, A., Piras, D., Duivenoorde, T., Lans, M., \& Sadeghian Marnani, H. (2019). Optimization of acoustic coupling for bottom actuated scattering based subsurface scanning probe microscopy. Review of Scientific Instruments, 90(7), [073705]. https://doi.org/10.1063/1.5097387

DOI:

10.1063/1.5097387

Document status and date:

Published: 01/07/2019

\section{Document Version:}

Publisher's PDF, also known as Version of Record (includes final page, issue and volume numbers)

\section{Please check the document version of this publication:}

- A submitted manuscript is the version of the article upon submission and before peer-review. There can be important differences between the submitted version and the official published version of record. People interested in the research are advised to contact the author for the final version of the publication, or visit the $\mathrm{DOI}$ to the publisher's website.

- The final author version and the galley proof are versions of the publication after peer review.

- The final published version features the final layout of the paper including the volume, issue and page numbers.

Link to publication

\section{General rights}

Copyright and moral rights for the publications made accessible in the public portal are retained by the authors and/or other copyright owners and it is a condition of accessing publications that users recognise and abide by the legal requirements associated with these rights.

- Users may download and print one copy of any publication from the public portal for the purpose of private study or research.

- You may not further distribute the material or use it for any profit-making activity or commercial gain

- You may freely distribute the URL identifying the publication in the public portal.

If the publication is distributed under the terms of Article 25fa of the Dutch Copyright Act, indicated by the "Taverne" license above, please follow below link for the End User Agreement:

www.tue.nl/taverne

Take down policy

If you believe that this document breaches copyright please contact us at:

openaccess@tue.nl

providing details and we will investigate your claim. 


\section{Optimization of acoustic coupling for bottom actuated scattering based subsurface scanning probe microscopy}

Cite as: Rev. Sci. Instrum. 90, 073705 (2019); https://doi.org/10.1063/1.5097387

Submitted: 25 March 2019 . Accepted: 19 June 2019 . Published Online: 16 July 2019

P. L. M. J. van Neer, B. Quesson, M. H. van Es (D), M. van Riel, K. Hatakeyama, A. Mohtashami, D. Piras,

T. Duivenoorde, M. Lans, and H. Sadeghian (D)

\section{ARTICLES YOU MAY BE INTERESTED IN}

Rosette-scan video-rate atomic force microscopy: Trajectory patterning and control design Review of Scientific Instruments 90, 073702 (2019); https://doi.org/10.1063/1.5098499

The interplay between drift and electrical measurement in conduction atomic force microscopy

Review of Scientific Instruments 90, 073701 (2019); https://doi.org/10.1063/1.5095647

Characterization of the phase sensitivity, visibility, and resolution in a symmetric neutron grating interferometer

Review of Scientific Instruments 90, 073704 (2019); https://doi.org/10.1063/1.5089588

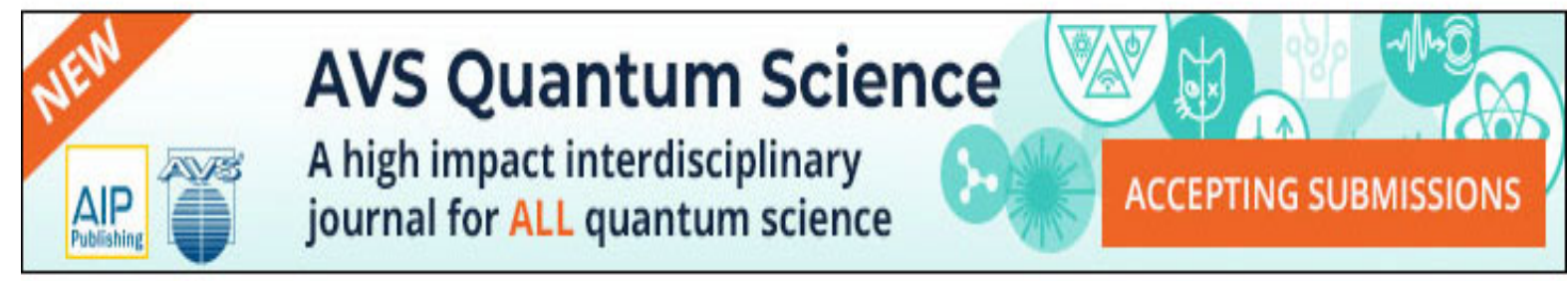




\title{
Optimization of acoustic coupling for bottom actuated scattering based subsurface scanning probe microscopy
}

Cite as: Rev. Sci. Instrum. 90, 073705 (2019); doi: 10.1063/1.5097387

Submitted: 25 March 2019 - Accepted: 19 June 2019 •

Published Online: 16 July 2019

P. L. M. J. van Neer, ${ }^{1,2, a)}$ B. Quesson, ${ }^{7}$ M. H. van Es, ${ }^{3}$ (D) M. van Riel, ${ }^{3}$ K. Hatakeyama, ${ }^{3}$ A. Mohtashami, ${ }^{3}$ D. Piras, ${ }^{3}$

T. Duivenoorde, ${ }^{3}$ M. Lans, ${ }^{3}$ and H. Sadeghian ${ }^{4,5}$ (D)

\begin{abstract}
AFFILIATIONS
${ }^{1}$ Department of Acoustics and Sonar, The Netherlands Organization for Applied Scientific Research (TNO), The Hague, Zuid-Holland 2597 AK, The Netherlands

${ }^{2}$ Acoustical Wavefield Imaging, ImPhys, Delft University of Technology, Delft, Zuid-Holland $2628 \mathrm{CJ}$, The Netherlands

${ }^{3}$ Department of Optomechatronics, The Netherlands Organization for Applied Scientific Research (TNO), Delft, Zuid-Holland 2628 $\mathrm{CK}$, The Netherlands

${ }^{4}$ Nearfield Instruments B.V., Rotterdam, Zuid-Holland, 3047 AT, The Netherlands

${ }^{5}$ Department of Mechanical Engineering, Eindhoven University of Technology, Eindhoven, Noord-Brabant 5612 AZ,

The Netherlands
\end{abstract}

a) Author to whom correspondence should be addressed: paul.vanneer@tno.nl

\begin{abstract}
The characterization of buried nanoscale structures nondestructively is an important challenge in a number of applications, such as defect detection and metrology in the semiconductor industry. A promising technique is Subsurface Scanning Probe Microscopy (SSPM), which combines ultrasound with Atomic Force Microscopy (AFM). Initially, SSPM was used to measure the viscoelastic contrast between a subsurface feature and its surrounding medium. However, by increasing the ultrasonic frequency to $>1 \mathrm{GHz}$, it has been shown that SSPM can also measure acoustic impedance based contrasts. At these frequencies, it becomes difficult to reliably couple the sound into the sample such that the AFM is able to pick up the scattered sound field. The cause is the existence of strong acoustic resonances in the sample, the transducer, and the coupling layer-the liquid layer used to couple the sound energy from the transducer into the sample-in combination with the nonlinearity of the tip-sample interaction. Thus, it is essential to control and measure the thickness of the coupling layer with nanometer accuracy. Here, we present the design of a mechanical clamp to ensure a stable acoustic coupling. Moreover, an acoustic method is presented to measure the coupling layer thickness in real-time. Stable coupling layers with thicknesses of $700 \pm 2 \mathrm{~nm}$ were achieved over periods of $2-4 \mathrm{~h}$. Measurements of the downmixed AFM signals showed stable signal intensities for $>1 \mathrm{~h}$. The clamp and monitoring method introduced here makes scattering based SSPM practical, robust, and reliable and enables measurement periods of hours.
\end{abstract}

Published under license by AIP Publishing. https://doi.org/10.1063/1.5097387

\section{INTRODUCTION}

The nondestructive characterization of buried nanoscale structures is an important challenge in different application areas. For the semiconductor industry application, examples are buried defect detection, ${ }^{1}$ lithography mask inspection, ${ }^{2}$ and metrology. ${ }^{3}$ In the application of biomedical diagnostics, example applications are the imaging of the internal structures of cells and tissues. ${ }^{4}$ A promising method is Subsurface Scanning Probe Microscopy (SSPM), which combines ultrasound with Atomic Force Microscopy (AFM). This technique has been shown to be capable of imaging buried nanostructures in various samples. ${ }^{5-8}$ Two different contrast mechanisms have been identified for SSPM in the literature:

(1) Viscoelasticity:

Here, the contrast is caused by the variation in the stressfield induced in the sample by the tip of the cantilever and the ultrasound field, due to local viscoelasticity changes in the 
sample (e.g., due to the presence of a subsurface feature). The ultrasound excitation typically has frequencies of 1-100 MHz. In this regime, wave propagation does not play a role; instead the ultrasound only serves to modulate the tip sample contact and thereby to excite the cantilever. Viscoelasticity based SSPM requires large indentation forces applied to the tip in order to extend the stress field in the sample and in that way probe the subsurface. Thus, the maximum detection depth of the method is limited to less than $1 \mu \mathrm{m}^{8,12}$ depending on the medium's compressive strength and the viscoelastic contrast between the subsurface feature and the surrounding medium.

(2) Scattering: $:^{14,15}$

Here, the contrast is caused by acoustic scattering due to differences in the acoustic impedance between buried features and the rest of the sample. Thus, the contrast depends on the stiffness and density differences between buried features and the surrounding sample medium. The scattered acoustic energy will eventually reach the sample surface, and cause a displacement that may be picked up using an AFM probe. As the scattered energy depends on the fourth power of the ultrasound frequency and the sixth power of the feature radius, ${ }^{15}$ ultrasonic frequencies $>1 \mathrm{GHz}$ are needed to detect subsurface features with nanometer or micrometer sizes. The static force on the cantilever can be low, as the penetration depth is independent of the said static force. Experimental results of features buried below $7 \mu \mathrm{m}$ of photoresist have been demonstrated in the literature.

While in this paper, we focus on the second contrast mechanism based on scattering, the existing literature is almost exclusively focused on the first contrast mechanism based on viscoelasticity by using much lower ultrasound frequencies. Even though many different excitation schemes and readout schemes have been proposed to excite the cantilever, such as ultrasonic force microscopy, atomic force acoustic microscopy ${ }^{7}$, or resonant difference-frequency atomic force ultrasonic microscopy, ${ }^{16}$ and different explanations for the origin of contrast are given for some of these techniques, they all typically employ ultrasound between 0.1 and $10 \mathrm{MHz}$. By virtue of the scaling of scattered energy by wavelength, as explained above, none of these methods provide contrast based on scattering of the ultrasound. The notable exception is the work by $\mathrm{Hu}$ et al. ${ }^{14}$ who employed ultrasound at $1 \mathrm{GHz}$ to visualize structures buried micrometers below a sample using scattering based contrast. In this work, we replicate and improve upon the experimental setup for scattering based SSPM as published by $\mathrm{Hu}$ et al. ${ }^{14}$ Figure 1 displays a schematic of such a bottom actuated scattering based SSPM setup.

Here, a signal consisting of a carrier frequency $\left(f_{c}\right)$ modulated using a modulation frequency $\left(f_{m}\right)$ is led to a piezotransducer consisting of a piece of piezomaterial and a delay line. The piezomaterial converts the electrical excitation into an acoustic (elastic) wave, which travels through the delay line, the coupling layer, and the sample. The role of the coupling layer is to maximize the transmission of acoustic energy from the delay line into the sample. The coupling layer generally consists of a liquid to ensure no gas bubbles are present in the acoustic beam, as the transmission of sound through a solid-gas/gas-solid interface is poor. Inside the sample, the acoustic wave is scattered by subsurface features. The scattered

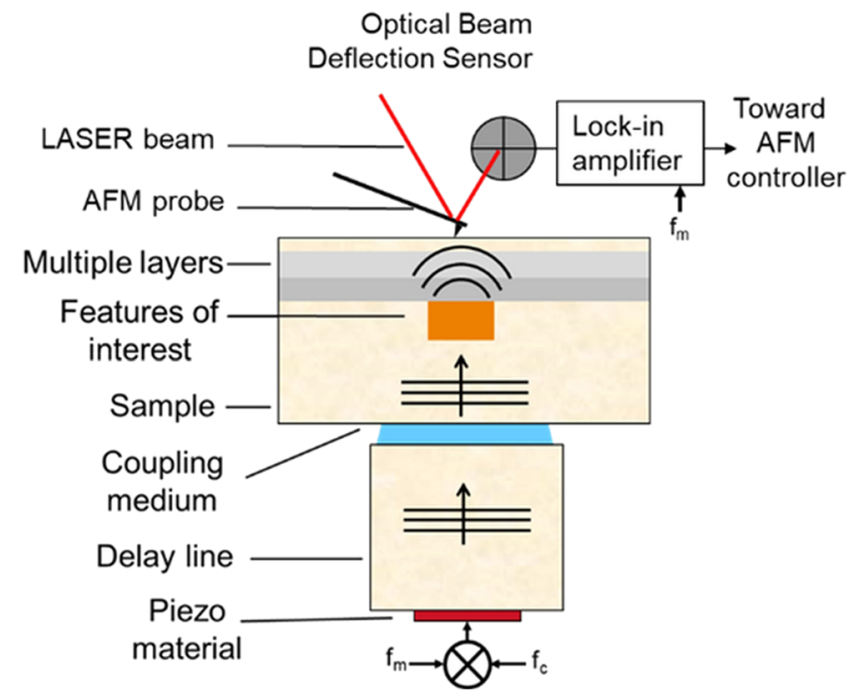

FIG. 1. Schematic of a bottom actuated scattering based SSPM setup.

wave then travels toward the sample top surface, and the resulting sample top surface displacement is picked up by an AFM probe. The contact between the tip and sample surface is described in the literature by means of Hertz theory. ${ }^{8,12,17}$ In Hertz theory, the relation between the contact force and the resulting tip indentation is nonlinear. Therefore, the acoustic frequencies that have been scattered toward the sample surface result in a displacement that is downmixed by the nonlinear tip-sample interaction. The analytical expansion of such mixed frequencies shows that the process generates a low frequency component signal at the chosen modulation frequency (or twice the modulation frequency depending on the modulation scheme). Details of the experimental setup relevant for the downmixing are reported in the work by van Es et al. ${ }^{8}$ Typically, the modulation frequency is chosen to be close to the contact resonance frequency of the cantilever $(0.1-1 \mathrm{MHz})$ to enhance the AFM sensitivity.

In the case of a $1 \mathrm{MHz}$ modulation frequency and a $1 \mathrm{GHz}$ carrier frequency, a thousand $1 \mathrm{GHz}$ cycles fit in the time length of one $1 \mathrm{MHz}$ cycle. For silicon, the wavelength at $1 \mathrm{GHz}$ is $\sim 9.7 \mu \mathrm{m}$ and the spatial length of a thousand $1 \mathrm{GHz}$ cycles is $\sim 9.7 \mathrm{~mm}$. As the thickness of the transducer and the sample combined is $\sim 1 \mathrm{~mm}$, during operation a complex acoustic interference pattern exists inside the transducer, coupling layer, and sample stack. Thus, frequencies exist where constructive interference occurs and frequencies exist where destructive interference occurs. At the carrier frequencies where constructive interference occurs, one may expect the highest sample surface displacements. However, the situation is more complex, as the coupling layer typically has a different acoustic impedance than the delay line and the sample. Because of this separate resonances exist in the transducer, the coupling layer, the sample, and all combinations thereof. These resonances overlap and interfere leading to a strong dependence of the sample surface displacement on the geometry and mechanical properties of the transducer, coupling layer, and sample. Thus, it is absolutely critical to select the carrier frequency producing a high sample surface displacement 
for the particular transducer/coupling layer/sample combination of interest. Moreover, the carrier frequency should be tracked over time or even better the geometry-and especially the liquid coupling layer-should be kept stable for the measurement duration (hours). Thus, there is also a need to monitor the thickness of the coupling layer over time. This can be done using acoustic measurements.

In this work, we present the design of a mechanical clamp system to ensure a stable acoustic coupling and thus to enable practical scattering based SSPM measurements. Moreover, an acoustic method is presented to measure the coupling layer thickness in realtime. Finally, scattering based SSPM measurements are presented.

\section{WAVE PROPAGATION}

\section{The coupling layer}

The coupling layer exists to maximize the transmission of acoustic energy from the transducer into the sample. To prevent the presence of gas bubbles in the acoustic beam (the transmission of sound through a solid-gas/gas-solid interface is poor), the coupling layer generally consists of a liquid. The ideal coupling layer liquid should

- have a low sound attenuation to minimize energy losses in the layer,

- have a low viscosity, such that the layer thickness may be rapidly minimized during mounting with minimal additional force,

- be nontoxic to keep the measurement protocols simple,

- be easily cleanable or leave no residues,

- have a reasonably low evaporation rate.

A number of liquids were evaluated as a coupling layer and demineralized water was selected. It has a low sound attenuation, ranging from $0.22 \mathrm{~dB} / \mu \mathrm{m}$ at $1 \mathrm{GHz}$ up $22 \mathrm{~dB} / \mu \mathrm{m}$ at $10 \mathrm{GHz}$. Its dynamic viscosity is $1.0016 \mathrm{mPa} \mathrm{s.}{ }^{19}$ It is nontoxic and leaves virtually no residue. Moreover, its evaporation rate is acceptably low.

\section{Wave propagation in the transducer-coupling layer-sample stack}

The dependency of the out-of-plane top sample surface displacement as a function of the thickness of the water coupling layer and the carrier frequency was investigated using the KLM model. ${ }^{20,21}$ The results are presented in Fig. 2. The acoustic stack consisted of a transducer made up of a $\mathrm{ZnO}$ piezolayer resonating at $1 \mathrm{GHz}$ and a $0.45 \mathrm{~mm}$ silicon delay line, a coupling layer consisting of water and a $0.7 \mathrm{~mm}$ silicon sample (see Fig. 1 for a schematic of the stack).

Figure 2(a) shows that the out-of-plane top sample surface displacement strongly depends on the thickness of the water layer and the carrier frequency. It appears that there are regularly spaced frequencies, where the sample surface displacement varies minimally. A zoom of Fig. 2(a) displayed in Fig. 2(b) shows that this is in fact not the case: even at the aforementioned frequencies, the surface displacement varies significantly as a function of the coupling layer thickness. The line graphs displayed in Fig. 2(c) indicate that the magnitude of the variation in out-of-plane top sample surface displacement at a particular carrier frequency can be 1-2 orders for different coupling layer thicknesses. This is caused by the
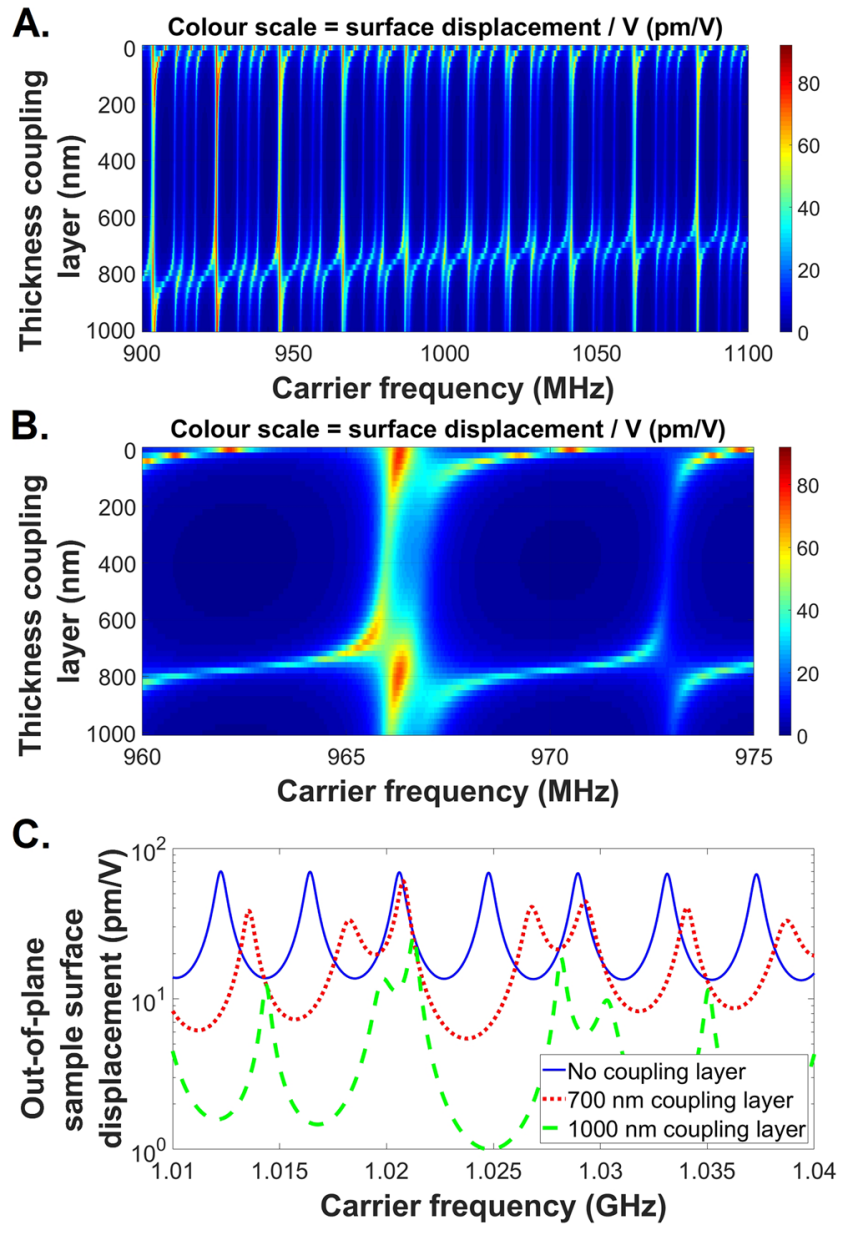

FIG. 2. (a) Simulated out-of-plane top sample surface displacement per excitation Voltage vs the thickness of the coupling layer and the carrier frequency. The colors indicate the out-of-plane top sample surface displacement in pm. This frequency response was calculated using the $1 \mathrm{D} \mathrm{KLM}$ model. ${ }^{20,21}$ The stack consisted of a transducer made up of a $\mathrm{ZnO}$ piezolayer resonating at $1 \mathrm{GHz}$ and a $0.45 \mathrm{~mm}$ silicon delay line, a coupling layer consisting of water and a $0.7 \mathrm{~mm}$ silicon sample (see Fig. 1). (b) zoom of the figure displayed in (a). (c) Simulated out-of-plane top sample surface displacement per excitation Voltage for three different coupling layer thicknesses: no coupling layer-solid blue line, $700 \mathrm{~nm}$-dotted red line, $1000 \mathrm{~nm}$-dashed green line.

constructive or destructive interference of the elastic waves propagating in the transducer-coupling layer-sample stack. The downmixed signal picked up by the AFM will vary even more $-2-3$ orders of magnitude-due to its nonlinear relation with the out-of-plane top sample surface displacement. The added variation due to the nonlinear tip-sample interaction depends on the chosen point of operation on the force-distance curve.

For practical coupling layer thicknesses $(>100 \mathrm{~nm}$, as there is always some warp present in the sample/transducer delay line), the simulation results showed that the minimum variation in coupling layer thickness to produce a factor 2 variation in out-of-plane sample surface displacement at a single carrier frequency was $20 \mathrm{~nm}$. The best way of achieving such a mechanical stability over a period 
of hours was deemed to be with mechanical contact between sample and transducer: the coupling layer should be made to be so thin that the peaks of the sample roughness rest on the peaks of the transducer roughness. In that case, the coupling layer thickness is determined by the warp and roughness of the transducer-coupling layer/coupling layer-sample interfaces. During an actual SSPM measurement the out-of-plane sample surface displacement would be maximized using a carrier frequency sweep. In that way, variations in sample properties, thicknesses, and the transducer-sample contact would be accounted for.

\section{DESIGN OF THE MECHANICAL CLAMP}

The aim of the mechanical clamp is to ensure a stable coupling layer with a minimal thickness for multiple hours. The minimal thickness is dictated by the warp and roughness of the transducer and sample interfaces. Although a single 2D SSPM scan takes approximately 10-15 min, the hour+long stability requirement comes from the fact that usually multiple measurements suitable for direct comparison are desired. Since the liquid for the coupling layer evaporates, a means of counteracting evaporation of the coupling layer liquid is also needed.

The stability requirement in the $\mathrm{z}$-direction is set to be $20 \mathrm{~nm}$, as the KLM simulations presented in the Wave Propagation section indicated that this would lead to a less than a factor of 2 variation in out-of-plane sample surface displacement. With the expected highest spatial wavelengths of the standing wave pattern to be in the range of $966 \mathrm{~nm}$ (at $10 \mathrm{GHz}$ ), a lateral (x, y) stability of the sample's position of $97 \mathrm{~nm}$ is deemed sufficient.

Bringing the top surface of the transducer and the bottom surface of the sample together requires alignment in three degrees of freedom: the out-of-plane rotations ( $\mathrm{Rx}$ and $\mathrm{Ry}$ ) and the out-ofplane displacement $(\mathrm{z})$ of the two surfaces need to be equal, with the $\mathrm{z}$-direction being along the surface normal. Should both surfaces be ideal surfaces, aligning them in $\mathrm{z}, \mathrm{Rx}$, and $\mathrm{Ry}$ results in perfect contact over the full sample/transducer surface. This is not the case, as imperfections in both surfaces exist: both the transducer as well as the sample surface possess a nonzero roughness and warp. As a result, the minimum coupling layer thickness that can be achieved is given by the quality of both surfaces.

In order to align both surfaces in $z, R x$, and Ry, three spatially separated forces are exerted along the surface normal, thus exerting a net alignment force in $\mathrm{z}$ and moment forces in $\mathrm{Rx}$ and $\mathrm{Ry}$. These forces are generated by three clamping fingers that press the sample down onto the transducer. The location of these fingers is chosen such that the three forces are exerted directly above the transducer surface, thus preventing tensile stresses in the sample, while leaving room for the AFM tip to access the sample; see Fig. 3. Samples could be coated with a soft layer, the plastic deformation of which is not desired. PMMA is chosen as the example soft material for the clamp design. At the end of each finger, a sphere is attached whose radius is chosen such that the maximum required clamp force does not result in plastic deformations of PMMA. While the maximum clamping force is such as to prevent plastic deformation on the sample's potentially soft top layer, the minimum clamping force is determined by the coupling medium's viscosity and evaporation rate. When the clamp presses the sample onto the transducer, it needs to expel the excess water from between the two surfaces. It was found that the higher the viscosity of the coupling medium, the slower the excess is drained, and the longer it takes for a stable situation, i.e., a situation where there exists mechanical contact between sample and transducer, to be reached. Moreover, if it takes too long for the excess coupling medium to be drained, the coupling medium evaporates, thus yielding insufficient or no coupling. Analytical calculations indicated that a minimum force is required to reach a stable situation in a timely fashion.

The sample's in-plane orientation $(\mathrm{Rz})$ and position $(\mathrm{x}$ and $\mathrm{y})$ is determined by an external manual positioning mechanism. By outfitting this mechanism with play between the end-effector and sample, the mechanism can be decoupled from the sample during measurements, thus preventing disturbances to be coupled into the sample/AFM during measurements. The stability of the lateral $(\mathrm{x}, \mathrm{y})$ position of the sample is determined by the friction between the transducer and sample. Care is taken that the lateral force that can be transferred onto the sample via the clamping fingers is substantially lower than the friction force between sample and transducer. Thus ensuring that the transducer-sample contact determines the xy-position stability. The limited size of the transducer top surface and the matched transducer delay line and sample (both silicon), results in a calculated lateral thermally induced displacement of $8.1 \mathrm{~nm} / 1^{\circ} \mathrm{C}$ temperature difference between the sample and transducer, where worst case temperature variations are expected to be of this magnitude. This results in significantly better lateral stability than the required $97 \mathrm{~nm}$.

\section{CONCEPT OF ACOUSTIC COUPLING LAYER THICKNESS MEASUREMENT}

The induced out-of-plane top sample surface displacement due to the scattering of ultrasound off subsurface features can be low. Moreover, the aforementioned surface displacement varies strongly as a function of the coupling layer thickness. To deal with these variations, the carrier frequency should be optimized such that the downmixed signal level at the modulation frequency remains high.
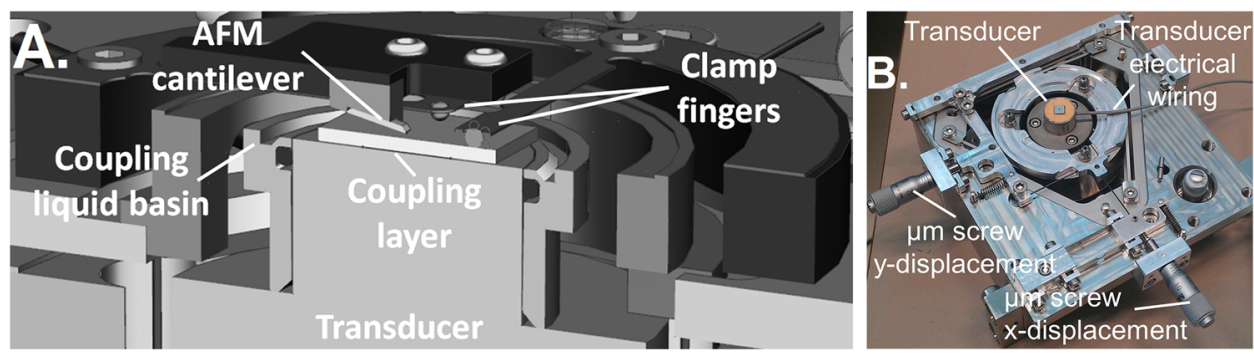

FIG. 3. (a) CAD drawing of a zoom of the clamp, the transducer-coupling layersample stack and the basin. (b) The realized clamp including transducer 
Therefore, it is important to monitor the thickness of the coupling layer in real-time. Here, the concept of an acoustic measurement method is discussed. The method uses the $\mathrm{GHz}$ transducer already present in the experimental setup (see Fig. 1), but utilizes short pulses rather than the continuous wave like excitation used in scattering based SSPM. Moreover, the transducer operates in pulse-echo mode rather than transmission mode.

Traditionally, the thickness of a layer is ultrasonically measured using the time of flight principle. The result is converted into a thickness using the wave speed. An example measurement is shown in Fig. 4(a), which displays a typical measured pulse-echo time trace recorded of the transducer-coupling layer-sample stack
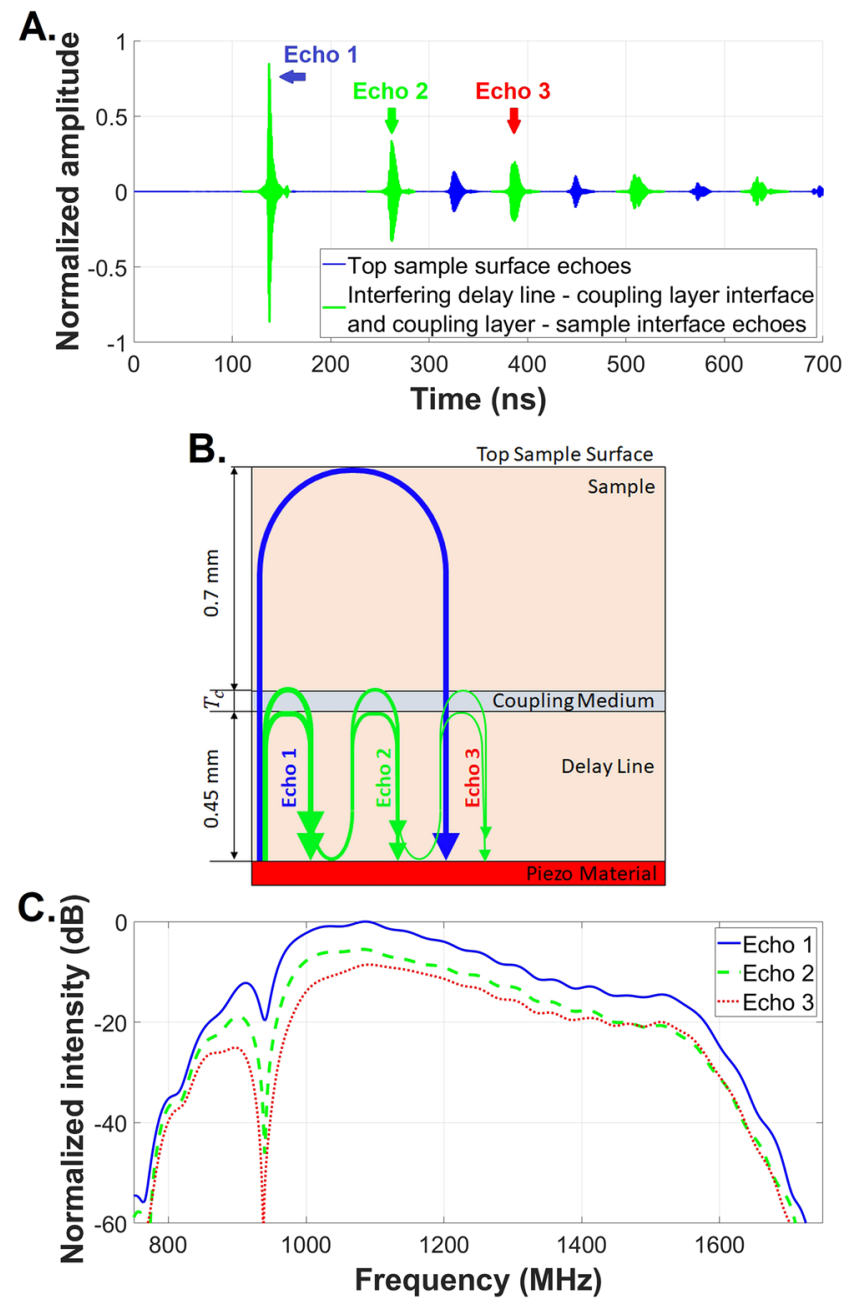

FIG. 4. (a) Example measurement of a pulse compressed recorded time trace. The setup with which these echoes have been recorded is described in the section "Experimental setup." The green line indicates the interfering echoes produced by the delay line-coupling layer interface and the coupling layer-sample interface. The first 3 echoes are indicated. The blue line indicates the echoes coming off the top sample surface. (b) A schematic figure detailing the layer stack and the travel path of the first four echoes arriving at the piezomaterial. (c) The amplitude spectrum of the Fourier transform of the three echoes indicated by the colored arrows shown in (a). The dip at $940 \mathrm{MHz}$ is indicative of the coupling layer thickness, which in this case was $798 \mathrm{~nm}$. [see Fig. 4(b) for a schematic image of the stack and the travel paths]. The green line indicates the interfering echoes produced by the delay line-coupling layer interface and the coupling layer-sample interface. The blue curve indicates echoes originating from the top sample surface. As is clearly visible in Fig. 4(a), the echoes of the delay line-coupling layer and coupling layer-sample interfaces interfere such that no separate echoes are visible. Thus, to estimate the thickness of the coupling layer, the time of flight method is not possible. The cause is the coupling layer thickness, which is in the order of one wavelength $(1.5 \mu \mathrm{m}$ at $1 \mathrm{GHz}$ in distilled water).

However, in the Fourier spectra of the interfering echoes produced by the delay line-coupling layer interface and the coupling layer-sample interface the aforementioned interference pattern produces distinct dips in their Fourier spectra [Fig. 4(c)]. These dips are indicative of frequencies where destructive interference occurs. As the wavelength is $\sim 1.5 \mu \mathrm{m}$ at frequencies of $\sim 1 \mathrm{GHz}$ and the transducer and the sample dimensions are $\gg 100 \mu \mathrm{m}$, this is an approximately $1 \mathrm{D}$ problem and diffraction effects may be neglected. In addition, the attenuation in water is at $0.22 \mathrm{~dB} / \mu \mathrm{m}$ at $1 \mathrm{GHz}$ very low, and may therefore be neglected as well. Furthermore, the particle displacements are expected to be low; hence, nonlinear wave propagation effects may also be neglected. A final assumption is that the acoustic impedance of the coupling layer is either lower or higher than the acoustic impedances of both the delay line and sample. Therefore, for a pulse-echo configuration the resonance dips may be expected at frequencies $f_{n}$

$$
f_{n}=\frac{n c_{c}}{2 T_{c}},
$$

with $n$ the resonance order, $T_{c}$ the thickness of the coupling layer, and $c_{c}$ the compressional wave speed in the coupling layer.

For two resonance orders $\left(f_{n+x}\right.$ and $\left.f_{n}\right)$ separated by a positive integer number of $x$ resonance orders, the coupling thickness can be derived independently of the resonance order

$$
T_{c}=\frac{x c_{c}}{2 \Delta_{f_{x}}}
$$

with $\Delta_{f_{x}}=f_{n+x}-f_{n}$.

In order to be able to measure a resonance dip for all relevant coupling layer thicknesses, the measurement system should have an upper frequency bandwidth limit $\left(f_{\text {upper }}\right)$ defined as

$$
f_{\text {upper }}>\frac{c_{c}}{2 T_{\min }}
$$

with $T_{\min }$ the minimal possible mean distance between the top of the transducer and the bottom of the sample as dictated by the warp and roughness of both aforementioned layers.

To ensure that no ambiguity exists on the resonance dip order, the lower frequency bandwidth $\left(f_{\text {lower }}\right)$ limit of the measurement system should be

$$
f_{\text {lower }} \leq f_{\text {upper }} / 3 \text {. }
$$

Note that it is assumed here that the sensitive frequency range of the piezotransducer is continuous and smooth.

In liquids, the compressional wave speed depends on the temperature and only slightly on the pressure. For distilled water at $20^{\circ} \mathrm{C}$ and at a pressure of 1 atmosphere, the compressional wave 
speed $\left(c_{w}\right)$ is $1483 \mathrm{~m} / \mathrm{s}^{23}$ and the temperature dependency of $c_{w}$ is $3 \mathrm{~m} / \mathrm{s} /{ }^{\circ} \mathrm{C}$ around $20^{\circ} \mathrm{C}$.

\section{EXPERIMENTAL SETUP}

\section{Setup description}

An arbitrary waveform generator (M8195A, Keysight, Santa Rosa, USA) generated electrical signals, which were amplified by a power amplifier (ZHL-2-8+, Mini-circuits, New-York, USA) and routed through a power splitter (ZFRSC-42-S+, Mini-circuits, NewYork, USA). The latter allowed for the monitoring and digitization of the transmitted signal on an oscilloscope (DSA 70804B, Tektronix, Beaverton, USA). The signal was routed through a circulator (PE83CR1013 and PE8432, Pasternack, Irvine, USA) to a piezoelectric transducer (custom designs with center frequencies of 1.1 and 3.1 GHz, Kibero, Saarbrücken, Germany). Return pulse-echo signals were routed by the circulator to the oscilloscope and digitized. The acoustic wave produced by the piezoelectric transducer travelled through the delay line, the coupling layer and the sample and displaced the sample surface. A schematic overview of the aforementioned part of the experimental setup is shown Fig. 5.

To measure the out-of-plane top sample surface displacement, an AFM (Dimension Icon, Bruker) was used. For this, the custom built clamp was mounted on the sample stage of the AFM with a dedicated embrace. The AFM probes (SCANASYST-AIR, Bruker Nano, Inc., Berlin, Germany) were operated in contact mode at set point forces of 10-30 nN. This force setting was chosen as a compromise between the desire to minimize stresses induced by the cantilever tip in the sample (to prevent possible viscoelastic contrast interfering with the scattering based contrast), the desire to maximize the nonlinearity of the tip-sample interaction, and the practical need to have robust measurements over a period of hours. The contact resonance frequency of the cantilever was determined using spectra of the thermal motion. For the scattering based SSPM measurements, the transducer was excited using an amplitude modulated excitation signal. It consisted of two high frequency sinusoids, the difference of which was close to the first order cantilever contact resonance. The downmixed signal induced by the out-of-plane sample surface displacement was picked up at the contact resonance frequency of

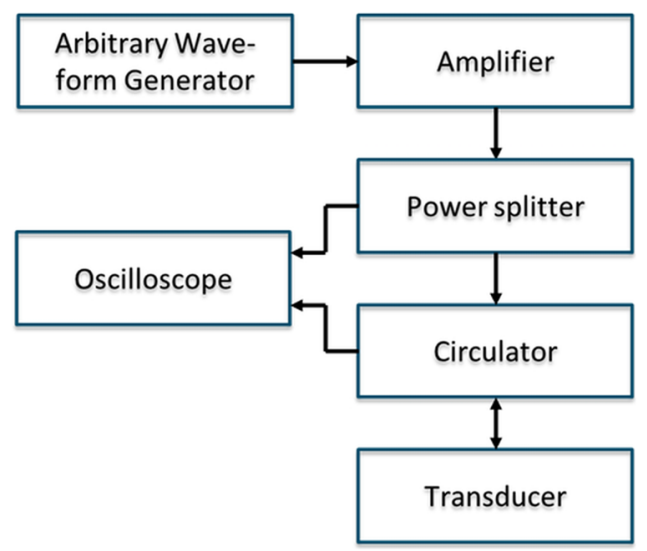

FIG. 5. Schematic overview of the experimental setup. the AFM cantilever and measured by the lock-in amplifier (UHFLI Lock-in Amplifier, Zurich Instruments, Zurich, Switzerland). Here, the reference signal was provided by the arbitrary waveform generator. A schematic overview of the experimental setup is shown in Fig. 1.

To measure the thickness of the coupling layer, the transducer was used in pulse-echo mode. The transmission signals consisted of linear frequency modulated (LFM) pulses with a pulse duration of 50 ns. For the $1.1 \mathrm{GHz}$ transducer, LFM pulses with a center frequency of $1.25 \mathrm{GHz}$ and $\mathrm{a}-3 \mathrm{~dB}$ (amplitude) bandwidth of $0.77 \mathrm{GHz}$ were used, whereas for the $3.1 \mathrm{GHz}$ transducer LFM pulses with a center frequency of $3 \mathrm{GHz}$ and a $-3 \mathrm{~dB}$ (amplitude) bandwidth of $5.7 \mathrm{GHz}$ were used. For each thickness estimate, 100 pulseecho traces were averaged. The pulse repetition frequency was $67 \mathrm{~Hz}$. Different pulse types were required for the scattering based SSPM and the coupling layer thickness measurements. Therefore, the latter measurements were interleaved with the former-the acoustic coupling layer thickness measurements were performed every minute or before and after each AFM scan (scan time typically 5-10 min).

The setup was located in a temperature controlled room limiting temperature variations to $<0.5^{\circ} \mathrm{C}$. Thus, the maximum absolute $c_{c}$ error of $1.5 \mathrm{~m} / \mathrm{s}$ led to an absolute thickness estimation error of $0.75 \mathrm{~nm}$.

The sample was a silicon slab with a size of $10 \times 10 \times 0.7 \mathrm{~mm}^{3}$.

\section{Signal processing-acoustic coupling layer thickness measurement}

A custom built signal processing algorithm was created to automatically extract the coupling layer thickness from the ultrasonic pulse-echo data. The algorithm performed the following operations on each acquired time trace:

1. Time windowing to suppress electrical crosstalk and reverberation.

2. Bandpass filtering.

3. Pulse compression to convert the linear frequency modulated pulses into short pulses and by that to enhance the signal-tonoise ratio.

4. Automatic extraction of the echoes originating from the delay line-coupling layer and the coupling layer-sample interfaces [see Fig. 4(a)] in two steps:

a. First echo detection using input geometry and thresholding.

b. Detection of subsequent 6 echoes using a search algorithm. Here, a forward acoustical model was fitted to the measurements and evaluated according to a maximum energy criterion.

5. Resonance frequency extraction in three steps:

a. A fast Fourier transform (FFT) was performed on each extracted echo [see an example in Fig. 4(b)].

b. The result was inverted to change the dips in the spectrum into peaks.

c. The result of $5 \mathrm{~b}$ was detrended.

d. The result of $5 \mathrm{c}$ was interpolated, where the oversampling factor was computed to match the required accuracy.

e. The frequencies of the resonances were extracted automatically using a threshold algorithm. Both the amplitude and 
phase information was used. The corresponding peak prominences (peak-to-valley ratios) were also saved as a quality metric.

6. The coupling layer thickness was estimated by combining multiple extracted resonance frequency estimations, where the resonances were weighted according to their prominence.

\section{Measurement protocol}

The measurement protocol consisted of the following steps:

(1) Mount the chosen transducer on the clamp.

(2) Mount the clamp on the sample stage of the AFM.

(3) Clean the top of the transducer and the bottom of the sample using isopropanol to remove micrometer sized particles, which could disrupt an optimal acoustic coupling.

(4) Mount the AFM probe.

(5) Align the cantilever with the transducer center and use this location as a reference.

(6) Add a droplet of water (typically $10 \mu \mathrm{l}$ ) on top of the transducer. The droplet volume is chosen to be slightly larger than the coupling layer volume as determined by the warp and roughness of the transducer top surface and sample bottom surfaces.

(7) Place the sample on top of the transducer top surface.

(8) Mount the top of the clamp to lock the sample in place.

(9) Start acoustic monitoring of coupling layer thickness.

(10) Wait until the coupling layer thickness stabilizes. Here, the criteria are: (1) a coupling layer thickness, which is equal or smaller than the warp/roughness of the top of the transducer surface plus the warp/roughness of the bottom side of the sample, and (2) the coupling layer thickness should vary less than $20 \mathrm{~nm}$ over $10 \mathrm{~min}$.

(11) Start the scattering based SSPM measurement.

\section{RESULTS AND DISCUSSION}

\section{Acoustic coupling layer thickness measurements}

The coupling layer thickness was continuously acoustically monitored during the scattering based SSPM measurements.

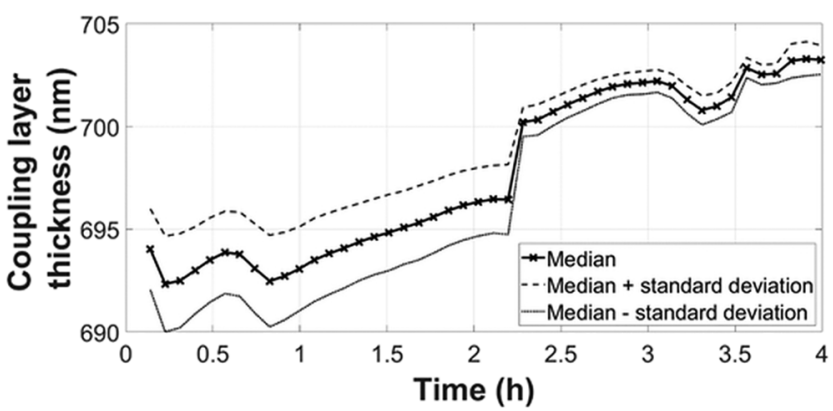

FIG. 6. Example measurement of the coupling layer thickness vs time. The black solid curve with crosses indicates the median, whereas the dotted and dashed black lines indicate the median \pm the standard deviation.
Figure 6 shows a typical example coupling layer thickness measurement as a function of time. The black solid curve with crosses indicates the median coupling layer thickness, whereas the dotted and dashed black lines indicate the median \pm the standard deviation.

The results displayed in Fig. 6 indicate that in this case a stable coupling layer thickness of 692-703 nm was achieved over a period of $4 \mathrm{~h}$. The standard deviation on the acoustic coupling layer thickness measurements was $0.7-2 \mathrm{~nm}$. The smooth upward trend in the data is likely caused by a temperature increase of the coupling layer liquid-a $1{ }^{\circ} \mathrm{C}$ change in temperature leads to a $1.5 \mathrm{~nm}$ apparent coupling layer thickness increase. Part of the temperature change was caused by the $2-3{ }^{\circ} \mathrm{C}$ temperature variation in the room during the day (the measurement took place in the morning), the remainder of the increase is likely due to local heating by the electrical equipment. A jump of about $4 \mathrm{~nm}$ was observed in the output coupling layer thickness estimation at a time of $\sim 2.2 \mathrm{~h}$. This corresponded to the moment in time where extra liquid was added to the basin to counteract evaporation. The aforementioned jump in the coupling layer thickness could be caused by the following:

(1) a small temperature difference between the added liquid and the transducer/sample. This led to a wave speed difference and therefore to a perceived change in coupling layer thickness.

(2) The sample shifted slightly with respect to the transducer due to the addition of the liquid, leading to a change in the measured coupling layer thickness.

The practical measurement time without filling the basin was approximately $10-15 \mathrm{~min}$. This was caused by the capillary action on the liquid between the sample and the transducer: the water evaporated on the outside of the coupling layer and was pulled to the outside by the capillary effect. When the basin was filled with liquid, the measurement time was extended up to $2+\mathrm{h}$.

\section{SPM measurements}

The simulations displayed in Fig. 2 predicted an out-of-plane sample surface displacement, which heavily depended on the choice of carrier frequency for a given coupling layer thickness. This was experimentally investigated by measuring the AFM response at a single spatial location while sweeping the carrier frequency. Figure 7 shows the AFM signal amplitude at the downmixed frequency as a function of the carrier frequency for two cases. Figure 7 (a) shows measurement results where the $1.1 \mathrm{GHz}$ transducer was used. The modulation frequency was set to $260 \mathrm{kHz}$. Figure 7 (b) shows measurement results where the $3.1 \mathrm{GHz}$ transducer was used. Here, the modulation frequency was set to $250 \mathrm{kHz}$. For both cases the carrier frequency was swept with $500 \mathrm{kHz}$ steps at a sweep rate of $8 \mathrm{~s}$ per step. The $\mathrm{GHz}$ transducer excitation Voltage was $\sim 6 \mathrm{~V}_{\mathrm{pp}}$.

The frequency spacing of the peaks is $\sim 8 \mathrm{MHz}$ for both the cases displayed in Fig. 7. Notice that there appear to be "extra" peaks, e.g., at 1.018 and $1.032 \mathrm{GHz}$ in Fig. 7(a) and at $2.816 \mathrm{GHz}$ in Fig. 7(b). The resonance pattern shown in Fig. 7(a) differs from the pattern calculated in Fig. 2(c). This is caused by the fact that the coupling layer thickness for the measurement in Fig. 7(a) differs from the one in Fig. 2(c). Moreover, the model used to generate the results displayed in Fig. 2(c) is 1 dimensional, whereas reality is $3 \mathrm{D}$. Since at 1 $\mathrm{GHz}$ the mode order is $>125$, small deviations due to the fact that the 
A.

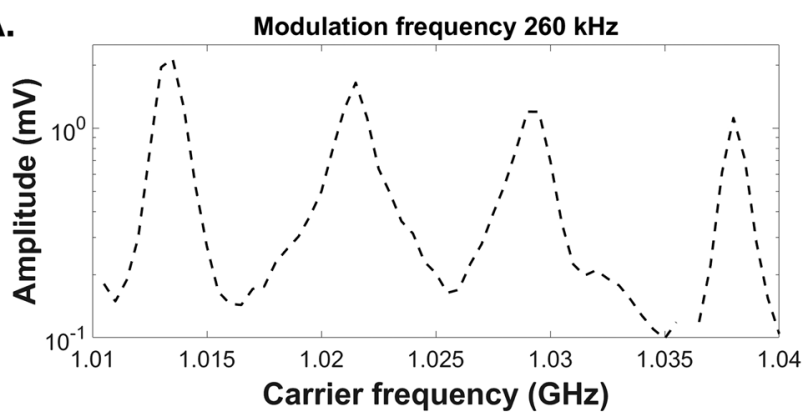

B.

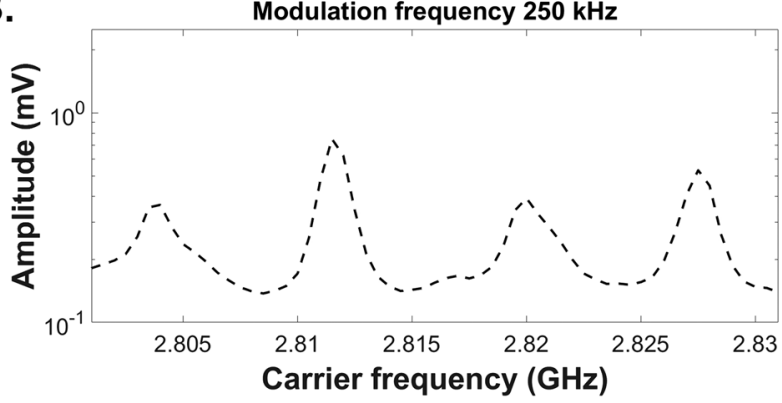

FIG. 7. AFM signal amplitude at the modulation frequency induced by sample surface displacement as a function of carrier frequency. (a) Measurement results obtained using a $1 \mathrm{GHz}$ transducer. The modulation frequency was set to $260 \mathrm{kHz}$. (b) Measurement results obtained using a $3.1 \mathrm{GHz}$ transducer. The modulation frequency was set to $250 \mathrm{kHz}$. For both cases, the carrier frequency was swept with $500 \mathrm{kHz}$ steps at a sweep rate of $8 \mathrm{~s}$ per step. The $\mathrm{GHz}$ transducer excitation Voltage was $\sim 6 \mathrm{~V}_{\mathrm{pp}}$

experiment is not truly $1 \mathrm{D}$ will lead to changes in the shape and amplitudes of the resonance peaks. The maximum peak to valley ratio is only in the range of 1 order of magnitude in the measurements, as these are limited by the noise.

To confirm that the coupling layer was stable sufficiently long such that multiple AFM scans could be made, the signal amplitude at the downmixed frequency was monitored over longer stretches of time. Figure 8 displays the downmixed signal amplitude vs the time. Figure 8(a) shows measurement results where the $1.1 \mathrm{GHz}$ transducer was used. The modulation frequency was set to $260 \mathrm{kHz}$ and the carrier frequency was set to $1021 \mathrm{MHz}$. Figure 8(b) shows measurement results where the $3.1 \mathrm{GHz}$ transducer was used. The modulation frequency was set to $250 \mathrm{kHz}$ and the carrier frequency was set to $2750 \mathrm{MHz}$.

In Fig. 8(a), the downmixed signal amplitude varied between 0.83 and $1.23 \mathrm{mV}$ over a period of slightly less than $1 \mathrm{~h}$, whereas in Fig. 8(b) the downmixed signal amplitude varied between 2.44 and $3.89 \mathrm{mV}$ over a period of a bit more than $1 \mathrm{~h}$. The aforementioned variance could be caused by not only the variations in the water layer thickness but also slight shifts in the contact resonance frequency due to drift in the probe contact force led by, for instance, variations in ambient temperature and control stability of the AFM setup. Although the signal-to-noise ratio varied slightly over this period, the variance in signal level is acceptably low for successful scattering based SSPM measurements.
A.

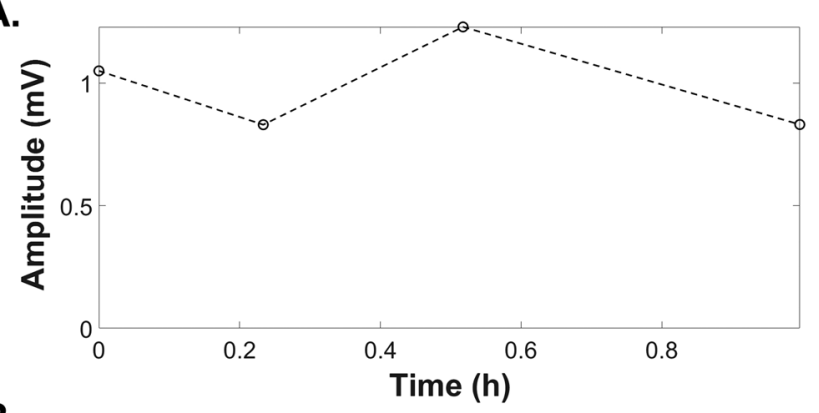

B.

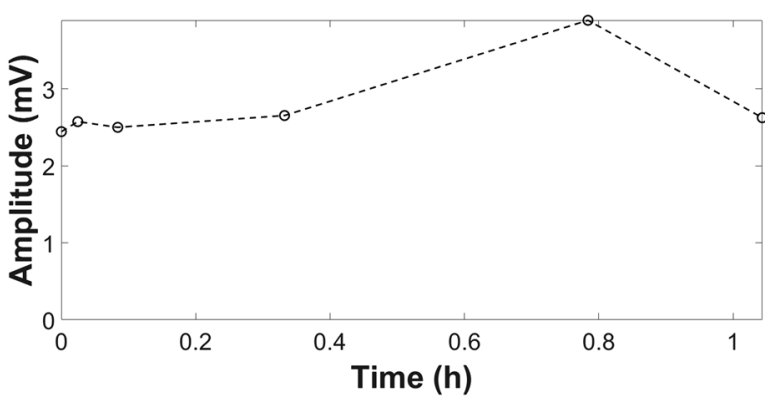

FIG. 8. (a) AFM signal amplitude at the modulation frequency of $260 \mathrm{kHz}$ as a function of time. The carrier frequency was $1.02 \mathrm{GHz}$. (b) AFM signal amplitude at the modulation frequency of $250 \mathrm{kHz}$ as a function of time. The carrier frequency was $2.75 \mathrm{GHz}$.

\section{CONCLUSION}

The acoustic coupling between the transducer and sample was optimized using a novel clamp design and monitored using the method described in this work. This made scattering based SSPM practical, robust, and reliable over a measurement period of hours.

\section{ACKNOWLEDGMENTS}

This research was supported by Early Research Program 3D Nanomanufacturing Instruments at the Netherlands Organization for Applied Scientific Research, TNO.

\section{REFERENCES}

1 O. D. Patterson, R. F. Hafer, X. Tang, and S. C. Lei, "The benefits of high landing energy for E-beam inspection," IEEE Trans. Semicond. Manuf. 29(4), 320-327 (2016).

${ }^{2}$ R. Jonckheere, D. van den Heuvel, F. Iwamoto, N. Stepanenko, A. Myers, M. Lamantia, A.-M. Goethals, E. Hendrickx, and K. Ronse, "Investigation of EUV mask defectivity via full-field printing and inspection on wafer," Proc. SPIE 7379, 73790R (2009).

${ }^{3}$ A. J. den Boef, "Optical wafer metrology sensors for process-robust CD and overlay control in semiconductor device manufacturing," Surf. Topogr.: Metrol. Prop. 4(2), 023001 (2016).

${ }^{4}$ L. Tetard, A. Passian, K. T. Venmar, R. M. Lynch, B. H. Voy, G. Shekhawat, V. P. Dravid, and T. Thundat, "Imaging nanoparticles in cells by nanomechanical holography," Nat. Nanotechnol. 3(8), 501-505 (2008).

${ }^{5}$ O. Kolosov, A. Briggs, K. Yamanaka, and W. Arnold, "Nanoscale imaging of mechanical properties by ultrasonic force microscopy (UFM)," in Acoustical Imaging, edited by P. Tortoli and L. Masotti (Springer, Boston, MA, 1996), Vol. 22, pp. 665-668. 
${ }^{6}$ J. P. Killgore, J. Y. Kelly, C. M. Staffort, M. J. Fasolka, and D. C. Hurley, "Quantitative subsurface contact resonance force microscopy of model polymer nanocomposites," Nanotechnology 22(17), 175706 (2011).

${ }^{7}$ D. C. Hurley, M. Kopycinska-Mueller, A. B. Kos, and R. H. Geiss, "Nanoscale elastic-property measurements and mapping using atomic force acoustic microscopy methods," Meas. Sci. Technol. 16(11), 2167-2172 (2005).

${ }^{8}$ M. H. van Es, A. Mohtashami, R. M. Thijssen, D. Piras, P. L. M. J. van Neer, and H. Sadeghian, "Mapping buried nanostructures using subsurface ultrasonic resonance force microscopy," Ultramicroscopy 184, 209-216 (2018).

${ }^{9}$ U. Rabe, S. Amelio, E. Kester, V. Scherer, S. Hirsekorn, and W. Arnold, "Quantitative determination of contact stiffness using atomic force acoustic microscopy," Ultrasonics 38(1), 430-437 (2000).

${ }^{10}$ Z. Parlak and L. F. Degertekin, in Acoustic Scanning Probe Microscopy, edited by F. Marinello, D. Passeri, and E. Savio (Springer-Verlag, Berlin, 2013), Chap. 15, pp. 417-436.

${ }^{11}$ K. Kimura, K. Kobayashi, K. Matsushige, and H. Yamada, "Imaging of $\mathrm{Au}$ nanoparticles deeply buried in polymer matrix by various atomic force microscopy techniques," Ultramicroscopy 133, 41-49 (2013).

${ }^{12}$ D. Piras and H. Sadeghian, "Analysis of contact stiffness in ultrasound atomic force microscopy: Three-dimensional time-dependent ultrasound modeling," J. Phys. D: Appl. Phys. 50(23), 235601 (2017).

${ }^{13}$ U. Rabe, M. Kopycinska-Müller, and S. Hirsekorn, Atomic Force Acoustic Microscopy, Acoustic Scanning Probe Microscopy (Springer, Berlin, Heidelberg, 2012).
${ }^{14} \mathrm{~S}$. Hu, C. Su, and W. Arnold, "Imaging of subsurface structures using atomic force acoustic microscopy at GHz frequencies," J. Appl. Phys. 109(8), 084324 (2011).

${ }^{15}$ R. S. C. Cobbold, Foundations of Biomedical Ultrasound (Oxford University Press, New York, 2007).

${ }^{16}$ S. A. Cantrell, J. H. Cantrell, and P. T. Lillehei, "Nanoscale subsurface imaging via resonant difference-frequency atomic force ultrasonic microscopy," J. Appl. Phys. 101, 114324 (2007).

${ }^{17} \mathrm{O}$. Kolosov and K. Yamanaka, "Nonlinear detection of ultrasonic vibrations in an atomic force microscope," Jpn. J. Appl. Phys., Part 2 32(8A), L1905-L1908 (1993).

${ }^{18}$ U. Rabe, M. Kopycinska-Mueller, S. Hirsekorn, and W. Arnold, "Evaluation of the contact resonance frequencies in atomic force microscopy as a method for surface characterization," Ultrasonics 40, 49-54 (2002).

${ }^{19}$ See https://wiki.anton-paar.com/en/water/ for Viscosity of water, Anton Paar.

${ }^{20}$ D. A. Leedom, R. Krimholtz, and G. Matthaei, "Equivalent circuits for transducers having arbitrary even- or odd-symmetry piezoelectric excitation," IEEE Trans. Sonics Ultrason, 18(3), 128-141 (1971).

${ }^{21}$ R. Krimholtz, "Equivalent circuits for transducers having arbitrary asymmetrical piezoelectric exctiation," IEEE Trans. Sonics Ultrason. 19(4), 427-436 (1972).

${ }^{22}$ G. J. Verbiest and M. J. Rost, "Beating beats mixing in heterodyne detection schemes," Nat. Commun. 6, 6444 (2015).

${ }^{23} \mathrm{~W}$. D. Wilson, "Speed of sound in distilled water as a function of temperature and pressure," J. Acoust. Soc. Am. 31, 1067 (1959). 